\title{
Consumo de drogas lícitas e ilícitas en Chile: resultados del estudio de 1998 y comparación con los estudios de 1994 y 1996
}

\author{
Ricardo Fuentealba, ${ }^{1}$ Francisco Cumsille, ${ }^{1,2}$ Juan Carlos Araneda ${ }^{1}$ \\ y Claudio Molina ${ }^{1,3}$
}

RESUMEN Se describen los principales resultados del Tercer Estudio Nacional de Consumo de Drogas realizado en Chile por el Consejo Nacional para el Control de Estupefacientes (CONACE) entre el 1 de septiembre de 1998 y el 15 de enero de 1999, que utilizó el mismo diseño metodológico de los estudios primero y segundo, realizados en 1994 y 1996, y que amplió la muestra a 31665 individuos que representaban a una población de 6940727 personas de 12 a 64 años de edad, de ambos sexos y de cinco niveles socioeconómicos, residentes en zonas urbanas de 62 comunas de las 13 regiones del país. Además, se comparan los resultados proporcionados por los tres estudios.

El estudio de 1998 muestra que 17,5\% de los chilenos han consumido alguna vez en la vida alguna de las tres drogas ilícitas de mayor uso en el país: marihuana $(16,8 \%)$, pasta base $(2,3 \%)$ y clorhidrato de cocaína (4,0\%). La prevalencia del consumo de cualquiera de las tres drogas durante el último año fue de 5,3\% y durante el último mes de 2,2\%, predominando una vez más el consumo de marihuana. Con respecto a las drogas lícitas, 28,4\% de los chilenos han consumido alguna vez en su vida ansiolíticos, 84,4\% alcohol y 71,9\% tabaco. La mayor parte de las personas que alguna vez consumieron drogas ilegales dejaron de hacerlo: 71,6, 64,1 y 66,8\% en el caso de la marihuana, de la pasta base de cocaína y del clorhidrato de cocaína, respectivamente. Para las drogas legales, estos porcentajes fueron menores: $55,5 \%$ para los ansioliticos, $16,0 \%$ para el alcohol y $34,5 \%$ para el tabaco.

El consumo de drogas lícitas e ilícitas fue varias veces mayor en los hombres que en las mujeres, excepto el de ansiolíticos, que fue tres veces mayor en el sexo femenino que en el masculino. El consumo fue más frecuente entre los 19 y 25 años de edad. El consumo de drogas ilícitas fue más frecuente en los niveles socioeconómicos más altos y el de drogas lícitas en los más bajos. El percentil 50 de la edad de inicio en el consumo de drogas se situó en los 17 años para el alcohol, en los 15 para el tabaco, en los 30 para los ansiolíticos, en los 17 para la marihuana, en los 20 para la pasta base de cocaína y en los 21 para el clorhidrato de cocaína.

La comparación de los resultados de los tres estudios realizados por el CONACE muestra que, tras el aumento del consumo de drogas lícitas registrado entre 1994 y 1996, se produjo una estabilización en 1998, mientras que para el consumo de drogas ilícitas hubo un ligero aumento entre 1996 y 1998, tras la ligera disminución observada entre 1994 y 1996.

1 Consejo Nacional para el Control de Estupefacientes, Santiago, Chile. Toda la correspondencia debe ser enviada a Ricardo Fuentealba, a la siguiente dirección postal: Consejo Nacional para el Control de Estupefacientes, Ahumada 11, Oficina 101, Santiago, Chile. Tlf. 562 5100800. Correo electrónico: rfuentealba@conace.cl

2 Escuela de Salud Pública, Universidad de Chile, Santiago, Chile.
La importancia que ha alcanzado en Chile, tanto para las políticas públicas (1) como para la intervención técnica,

3 Centro de Perfeccionamiento, Experimentación e Investigaciones Pedagógicas, Santiago, Chile. el poder contar con un diagnóstico periódico sobre el estado del consumo de drogas en el país ha hecho que este tipo de investigaciones proporcione información cada vez más detallada y específica tanto del consumo y dependen- 
cia como del ámbito geográfico en el que ocurre. Así, los tres estudios nacionales sobre el consumo de drogas realizados por el Consejo Nacional para el Control de Estupefacientes (CONACE) en 1994, 1996 y 1998, además de ir ampliando las muestras, han ido restringiendo las unidades regionales de análisis: tres grandes zonas geográficas censales en 1994 (2), las 13 regiones del país en 1996 (3) y la división administrativa más pequeña, la comuna, en 1998 (4). De este modo, Chile cuenta desde 1994 con mediciones bienales del consumo y dependencia de drogas lícitas e ilícitas a nivel nacional, lo cual ha permitido medir tendencias y orientar las políticas y estrategias de intervención relacionadas con el consumo de drogas. La experiencia chilena es comparable con la de muy pocos países latinoamericanos. Solo México y Colombia han realizado estudios nacionales con la misma periodicidad que Chile. Sin embargo, este último es el único país que cuenta con información del nivel de población más local.

De acuerdo con los resultados del II Estudio de Salud Mental y Consumo de Sustancias Psicoactivas (5), realizado en Colombia en 1997, la marihuana es, al igual que en Chile, la droga ilícita más consumida en ese país, con una prevalencia de $9,2 \%$ a lo largo de toda la vida y de $4,1 \%$ en el último año, y con una tasa de abandono del consumo de 5,1\%. Los hombres consumen marihuana en Colombia en una proporción bastante más alta que las mujeres, siendo la relación de 7:1. En Chile esta proporción es menor (3:1). Mientras que en Chile el grupo de edad con mayor consumo de marihuana es el de 19 a 25 años, en Colombia este es el de 25 a 29 años. Sorprende el hecho de que el segundo grupo con mayor consumo de marihuana en Colombia sea el de los mayores de 60 años.

Los objetivos del Tercer Estudio Nacional de Consumo de Drogas, realizado en Chile en 1998, consistieron en: 1) determinar la prevalencia e incidencia del consumo de drogas lícitas e ilícitas y las tasas de dependencia y de abandono del consumo en el país, y 2) analizar su distribución por comunas y según diferentes factores y características del indi- viduo, su familia y el entorno. Además, como los tres estudios nacionales sobre el consumo de drogas realizados hasta ahora en Chile han utilizado, básicamente, el mismo diseño metodológico, esto ha permitido comparar la evolución de las principales tasas estimadas en los años 1994, 1996 y 1998. En esta publicación solo se presenta información acerca del consumo de drogas según tres variables sociodemográficas y de su evolución en el tiempo.

\section{MATERIALES Y MÉTODOS}

En general, el diseño del estudio de 1998 fue similar al de los estudios de 1994 y 1996. Las variables dependientes analizadas en esta publicación fueron las tasas de prevalencia, incidencia y abandono del consumo de las principales drogas lícitas e ilícitas consumidas en Chile, con sus intervalos de confianza de $95 \%$. En el primer grupo se incluyeron el alcohol, el tabaco y los ansiolíticos, y en el segundo la marihuana, la pasta base de cocaína y el clorhidrato de cocaína. En relación con las drogas ilícitas, la expresión "cualquier droga" se refiere al consumo de al menos una de las tres. Frecuentemente, una persona consume más de una de ellas, por lo que las tasas de prevalencia del consumo de las distintas drogas no se pueden sumar. Las tasas de prevalencia se calcularon para diferentes períodos de tiempo: toda la vida (PV), último año (PA) y último mes (PM); los datos se expresan como porcentajes de personas que habían consumido drogas en el período señalado, obtenidos con la pregunta: “¿Cuándo fue la última vez que Ud. consumió?". El abandono del consumo se refiere a las personas que, habiendo consumido alguna vez en su vida, han dejado de hacerlo hace más de un año, contado desde el momento de la realización de la encuesta. La incidencia corresponde a los casos nuevos, es decir, las personas que iniciaron el consumo durante el último año, obtenidos con la pregunta: “¿Cuándo fue la primera vez que Ud. consumió?".

Estos datos fueron analizados en función de tres variables sociodemo- gráficas: sexo, edad y nivel socioeconómico. Se consideraron cinco grupos de edad (12 a 18, 19 a 25, 26 a 34,35 a 44 y 45 a 64 años) y cinco niveles socioeconómicos (alto, medio-alto, medio, medio-bajo y bajo). El nivel socioeconómico se estableció con una adaptación del índice de Graffar, validada anteriormente en la población chilena (6). Las variables utilizadas fueron la ocupación e instrucción del cabeza de familia, la fuente de ingresos familiares, la calidad de la vivienda y la calidad del barrio. Cada pregunta fue puntuada en una escala de 1 a 5 , de forma que la puntuación total varía entre 5 y 25 puntos. Las puntuaciones de 5 a 9 correspondieron al nivel alto, las de 10 a 13 al nivel medio-alto, las de 14 a 17 al nivel medio, las de 18 a 21 al nivel medio-bajo y las de 22 a 25 al nivel bajo.

El universo estudiado correspondió a la población de 12 a 64 años de edad con residencia habitual en viviendas particulares del área urbana de 62 comunas representativas del país. Se utilizó una muestra probabilística y sin reemplazo, definida en tres etapas y estratificada por comunas, a partir de una actualización del marco muestral a nivel comunal. Este marco muestral se encuentra dividido en comunas, que a su vez están divididas en secciones o conglomerados geográficos, según el Censo de Población y Vivienda de 1992. Esta información es actualizada periódicamente por el Instituto Nacional de Estadísticas (INE).

Las unidades de muestreo en cada etapa correspondieron a: 1) los conglomerados geográficos o secciones, 2) las viviendas particulares y 3 ) las personas de 12 a 64 años residentes en las viviendas seleccionadas. La muestra teórica incluyó 1726 conglomerados geográficos como unidades de la primera etapa, 41784 viviendas particulares como unidades de la segunda etapa y 41784 personas de 12 a 64 años de edad como unidades de la tercera etapa. En este tamaño muestral se incorporó un 33\% de las viviendas y personas adicionales a la muestra necesaria calculada, con el fin de cubrir eventuales pérdidas.

La fórmula usada para obtener el tamaño de la muestra fue: $m=\left(Z^{2} \cdot P Q /\right.$ 
$\left.E_{a}\right)$. Deff $=31490$, donde $m$ es el tamaño de la muestra; $Z^{2}$ el coeficiente absoluto que representa un nivel de confianza de $95 \%$ para una distribución normal $(1,96) ; P Q$ la varianza máxima, asumiendo que $P=Q=0,5 ; E_{n}$ el error absoluto de la variable estudiada, y Deff el efecto del diseño, considerando que se trata de una muestra obtenida en tres etapas.

Del total de viviendas, se descartaron 6 286: 3404 por tratarse de viviendas no particulares, demolidas, deshabitadas, no localizadas o de uso comercial u otro, y 2882 debido a que no vivían en ellas personas de 12 a 64 años, a que las personas seleccionadas estuvieron hospitalizadas durante el período de encuesta o a que no podían responder por discapacidad. De esta forma, la muestra potencialmente encuestable fue de 35498 viviendas.

En la primera etapa de muestreo se realizó una selección de los conglomerados geográficos con probabilidad proporcional al número de viviendas incluidas en cada una. En la segunda etapa se seleccionaron, con probabilidades iguales, las viviendas dentro de cada unidad de la primera etapa. En la tercera etapa, en cada vivienda seleccionada, se eligió, con igual probabilidad y mediante una selección probabilística según el método de Kish, a una persona de 12 a 64 años de edad. Mediante una entrevista dirigida, se encuestó a una persona de cada una de las viviendas particulares en las que fue posible aplicar la encuesta. A cada persona seleccionada se le explicaron las características del estudio y se le informó de la confidencialidad de sus respuestas y del anonimato de su participación. Se logró estrevistar a 31665 residentes en las 35498 viviendas potencialmente encuestables $(89,2 \%)$. Este procedimiento permitió generar muestras independientes entre sí, representativas de 62 comunas del país.

En el cuadro 1 se presentan los errores de muestreo para diferentes proporciones posibles de un determinado atributo, considerando una muestra efectiva de 31665 personas. De esta tabla se desprende, por ejemplo, que si el consumo de marihuana de la pobla-

CUADRO 1. Errores muestrales absolutos para diferentes porcentajes de un atributo

\begin{tabular}{lccccccccc}
\hline Porcentaje del atributo & 0,5 & 1,0 & 3,0 & 5,0 & 10,0 & 20,0 & 30,0 & 40,0 & 50,0 \\
Error muestral & 0,08 & 0,12 & 0,20 & 0,26 & 0,36 & 0,48 & 0,55 & 0,58 & 0,60 \\
\hline
\end{tabular}

ción encuestada en el último año fue de $10 \%$, el error atribuible a la muestra varía entre 9,64 y $10,36 \%$. Es importante destacar que los errores de muestreo descritos corresponden a estimaciones obtenidas tras la realización del estudio y, por lo tanto, están basados en los resultados del mismo, de tal forma que deben considerarse como valores de referencia a la hora de calcular el intervalo de confianza para alguna característica deseada.

De acuerdo con el diseño de la investigación, corresponde aplicar a cada persona seleccionada un factor de expansión que depende del número de personas de 12 a 64 años que tiene la vivienda, del número de viviendas que tiene el conglomerado geográfico y del número de conglomerados que tiene la ciudad. Los factores de expansión incluyen un ajuste de población de las personas de 12 a 64 años de edad en cada una de las comunas para aumentar la precisión de las estimaciones. Este factor se puede interpretar como el número de personas de la población que representa una persona de la muestra.

El formulario de la encuesta fue confeccionado para este estudio por el equipo investigador, considerando la experiencia recogida en los estudios de 1994 y 1996 y otros estudios disponibles (7-10). Además, se incluyeron las sugerencias recibidas desde las instituciones públicas consultadas. La encuesta se realizó entre el 1 de septiembre de 1998 y el 15 de enero de 1999. La empresa contratada para realizar el trabajo de campo (SKOPUS) supervisó $11924(28,5 \%)$ de las viviendas de la muestra original, independientemente de la supervisión realizada por la Secretaría Ejecutiva del CONACE en toda la fase del trabajo de campo. La supervisión del CONACE, que alcanzó a 7345 viviendas $(17,6 \%$ de la muestra teórica), se realizó mediante la participación de 100 profesionales, funcionarios municipales de las 62 comunas estudiadas, que fueron destacados en comisión de servicio desde sus municipios por solicitud de $\mathrm{CO}$ NACE. Para cumplir cabalmente con este objetivo, el equipo investigador realizó un programa de capacitación en distintos lugares del país, dos meses antes de iniciar la fase de ejecución de las encuestas. Los cursos tuvieron una duración de dos días y se realizaron en conjunto con el INE y la empresa encargada del trabajo de campo; participaron en ellos los supervisores municipales, los encuestadores y los supervisores de la empresa. Cada supervisor municipal debió emitir informes semanales sobre su tarea de campo y comunicar los problemas propios de esta etapa del estudio. Esta estrategia permitió conocer oportunamente las dificultades y resolverlas mediante instrucciones específicas a los supervisores; cuando fue necesario, un miembro del equipo investigador visitó y resolvió directamente las dificultades en la comuna. La evaluación global de la supervisión tuvo un seguimiento permanente en la sede del CONACE, mediante reuniones semanales con la empresa contratada.

\section{RESULTADOS}

\section{Descripción de la muestra}

La muestra efectiva de 31665 personas encuestadas $(46,0 \%$ del sexo masculino y $54,0 \%$ del sexo femenino) constituyó $89,2 \%$ de la muestra potencialmente encuestable y representó a un universo de 6940727 individuos de 12 a 64 años de edad con residencia habitual en viviendas particulares del área urbana de 62 comunas representativas del país. En el cuadro 2 se presentan las características generales de la muestra, según las tres variables sociodemográficas consideradas. 
CUADRO 2. Distribución de la muestra según el sexo, la edad y el nivel socioeconómico. Chile, 1998

\begin{tabular}{|c|c|c|c|}
\hline \multirow[b]{2}{*}{ Variable } & \multicolumn{2}{|c|}{ Muestra $(n=31665)$} & \multirow{2}{*}{$\begin{array}{c}\text { Población } \\
\text { representada } \\
(N=6940727)\end{array}$} \\
\hline & No. & $\%$ & \\
\hline \multicolumn{4}{|l|}{ Sexo } \\
\hline Masculino & 14295 & 46,0 & 3193287 \\
\hline Femenino & 17370 & 54,0 & 3747440 \\
\hline \multicolumn{4}{|l|}{ Edad (años) } \\
\hline $12-18$ & 4689 & 17,9 & 1241842 \\
\hline $19-25$ & 4766 & 16,9 & 1171790 \\
\hline $26-34$ & 6882 & 20,1 & 1394931 \\
\hline $35-44$ & 6843 & 20,7 & 1438862 \\
\hline $45-64$ & 8485 & 24,4 & 1693302 \\
\hline \multicolumn{4}{|c|}{ Nivel socioeconómico } \\
\hline Alto & 2439 & 9,6 & 666096 \\
\hline Medio-alto & 7771 & 25,0 & 1732566 \\
\hline Medio & 11507 & 35,4 & 2456624 \\
\hline Medio-bajo & 8504 & 26,0 & 1804411 \\
\hline Bajo & 1444 & 4,0 & 281030 \\
\hline
\end{tabular}

\section{Consumo de drogas ilícitas}

Tasas de prevalencia, incidencia y abandono del consumo. Las tasas de PV, PA, PM, incidencia y abandono del consumo de drogas ilícitas se presentan en el cuadro 3 , junto con sus intervalos de confianza de 95\%. La PV del consumo de cualquiera de las tres drogas consideradas fue de 17,5\%. La PV del consumo de marihuana fue más de cuatro veces mayor que la del consumo de clorhidrato de cocaína y esta casi el doble que la del consumo de pasta base de cocaína. La PA del consumo de cualquiera de las tres drogas fue de $5,3 \%$. La PA del consumo de marihuana fue casi seis veces mayor que la del consumo de pasta base de cocaína y casi cuatro veces mayor que la del consumo de clorhidrato de cocaína. La PM del consumo de cualquiera de las tres drogas fue de $2,2 \%$. Una vez más, la cifra más elevada correspondió a la marihuana, siendo cerca de cinco veces superior a la del clorhidrato de cocaína y a la de la pasta base de cocaína.

La incidencia del consumo de cualquiera de estas tres drogas en el año anterior a la realización de la encuesta

CUADRO 3. Tasas de prevalencia, incidencia y abandono del consumo de las principales drogas ilícitas ${ }^{a}$. Chile, 1998

\begin{tabular}{lccccc}
\hline Tipo de droga & PVb & $\mathrm{PA}^{\mathrm{c}}$ & $\mathrm{PM}^{\mathrm{d}}$ & Incidencia $^{\mathrm{e}}$ & $\begin{array}{c}\text { Abandono del } \\
\text { consumo }^{\dagger}\end{array}$ \\
\hline Marihuana & 16,77 & 4,73 & 1,95 & 1,85 & 71,57 \\
Pasta Base & $(16,12-17,42)$ & $(4,38-5,08)$ & $(1,74-2,17)$ & $(1,60-2,10)$ & $(69,97-73,37)$ \\
& 2,27 & 0,81 & 0,37 & 0,35 & 64,10 \\
Cocaína & $(2,06-2,48)$ & $(0,73-0,95)$ & $(0,27-0,47)$ & $(0,27-0,43)$ & $(59,57-68,73)$ \\
& 4,02 & 1,32 & 0,41 & 0,70 & 66,79 \\
Cualquiera & $(3,71-4,33)$ & $(1,16-1,48)$ & $(0,31-0,51)$ & $(0,58-0,82)$ & $(63,38-70,20)$ \\
& 17,45 & 5,31 & 2,23 & 1,95 & - \\
\hline
\end{tabular}

a Todos los datos se presentan como porcentajes, con sus intervalos de confianza de $95 \%$.

b $\mathrm{PV}=$ prevalencia a lo largo de toda la vida.

c $P A=$ prevalencia en el año anterior a la realización de la encuesta.

d $\mathrm{PM}=$ prevalencia en el mes anterior a la realización de la encuesta.

e Incidencia en el año anterior a la realización de la encuesta.

f Abandono del consumo más de un año antes de la realización de la encuesta.

según el nivel socioeconómico. E consumo de drogas ilícitas afectó a todos los grupos socioeconómicos, con una distribución en forma de "W". Así, la mayor PV correspondió a los niveles socioeconómicos alto y bajo, se- 
guidos del nivel medio $\mathrm{y}$, por último, de los niveles medio-alto y mediobajo. En todo caso, las variaciones entre los diferentes grupos fueron escasas: poco más de $3 \%$ entre el grupo de mayor consumo (el de alto nivel socioeconómico) y el de menor consumo (el de nivel socioeconómico mediobajo) de cualquiera de las tres drogas ilícitas estudiadas.

PA según el sexo. Como ocurrió con la $\mathrm{PV}$, el consumo de cualquier droga ilícita en el último año mostró una diferencia apreciable entre los sexos. En efecto, el consumo de cualquier droga en el sexo masculino fue más del triple del observado en el sexo femenino. Las mayores diferencias se observaron en los consumos de pasta base de cocaína y clorhidrato de cocaína, que fueron, respectivamente, más de seis y casi cinco veces mayores en los hombres que en las mujeres; el de marihuana fue más de tres veces mayor en los hombres.

$P A$ según la edad. Se mantuvo la distribución observada para la PV. Así, la mayor PA del consumo de cualquiera de las tres drogas correspondió al grupo de 19 a 25 años. El consumo de drogas en el grupo de 45 a 64 años de edad fue 27 veces menor que el observado en el grupo de 19 a 25 años. La tendencia a la disminución del consumo a medida que aumenta la edad permite proyectar una tendencia hacia la abstinencia más allá de los 64 años de edad. En general, esta tendencia se mantuvo al considerar separadamente el consumo de cada una de las tres drogas.

$P A$ según el nivel socioeconómico. Este análisis mostró una distribución heterogénea según el tipo de droga. En el caso del consumo de cualquiera de las tres drogas ilícitas, los mayores valores correspondieron al nivel socioeconómico bajo y los menores al medio-alto. En los otros tres niveles socioeconómicos se observaron valores semejantes. El nivel socioeconó- mico bajo concentró las mayores tasas de consumo de marihuana y pasta base de cocaína, mientras que la mayor tasa de consumo de clorhidrato de cocaína correspondió al nivel medio.

PM según el sexo. La PM del consumo de drogas ilícitas fue cinco veces mayor en el sexo masculino que en el femenino. La relación se mantuvo al analizar separadamente el consumo de cada una de las tres drogas. Así, el consumo de clorhidrato de cocaína fue casi siete veces mayor en el sexo masculino que en el femenino, el de pasta base más de cinco veces mayor y el de marihuana casi cinco veces mayor.

PM según la edad. Las cifras más elevadas correspondieron a los tres primeros grupos de edad, que van de los 12 a los 34 años, y en particular al de 19 a 25 años, manteniéndose la tendencia observada con la PV y la PA. El consumo de drogas en los grupos de mayor edad fue varias veces inferior al de los menores de 35 años; así, por ejemplo, el consumo del grupo de 19 a 25 años fue 24 veces mayor que el del grupo de 45 a 64 años. Esta distribución se mantuvo para cada una de las drogas con variaciones menores en los distintos grupos de edad, aunque las mayores frecuencias se registraron siempre entre los 19 y 25 años. En el grupo de 45 a 64 años, la cantidad de consumidores tendió a ser nula.

PM según el nivel socioeconómico. La mayor PM correspondió al nivel socioeconómico bajo y la menor al nivel medio-alto; en los niveles alto y medio se registraron valores intermedios. Esta tendencia se mantuvo al analizar el comportamiento del consumo de marihuana, pero se observaron variaciones con respecto al consumo de las otras dos drogas. Así, mientras que el consumo de pasta base de cocaína disminuyó a medida que aumentaba el nivel socioeconómico, el consumo de clorhidrato de cocaína fue mayor en los niveles socioeconómicos medio, medio-alto y bajo, por este orden.
Edad de inicio del consumo. La edad de inicio del consumo fue diferente según la droga. Considerando el percentil 50 de la distribución, es decir la edad en que $50 \%$ de los consumidores dicen haber iniciado ya el consumo, la edad de inicio del consumo de marihuana fue a los 17 años; el consumo de las otras dos drogas se inició más tardíamente: a los 21 años el de clorhidrato de cocaína y a los 20 el de pasta base de cocaína. Considerando el percentil 5, el consumo de marihuana se inició a los 13 años, el de pasta base a los 14 y el de clorhidrato de cocaína a los 15. Para el percentil 95, las edades correspondientes fueron de 26, 37 y 37 años, respectivamente.

\section{Consumo de drogas lícitas}

Tasas de prevalencia, incidencia y abandono. Las tasas de PV, PA, PM, incidencia y abandono del consumo de drogas lícitas se reflejan en el cuadro 4. La PV del consumo de alcohol fue similar a la del consumo de tabaco y casi cuatro veces mayor que la del consumo de ansiolíticos. La mayor PA correspondió al alcohol, siendo 1,5 veces mayor que la del tabaco y casi seis veces mayor que la de los ansiolíticos. Igual que en el caso de las drogas ilícitas, estos valores disminuyeron al considerar el consumo en el mes anterior; la mayor disminución (casi a la mitad) correspondió a los ansiolíticos.

Con respecto a la incidencia del consumo de estas drogas en el año anterior al estudio, la cifra más elevada correspondió al alcohol, con una tasa más de tres veces mayor que la de los ansiolíticos y más de dos veces mayor que la del tabaco. La mayor tasa de abandono del consumo correspondió a los ansiolíticos, seguidos del tabaco y del alcohol.

Prevalencia según las variables sociodemográficas. A continuación se analizan las variaciones de la PA y PM del consumo de drogas lícitas en función de las tres variables sociodemográficas. 
CUADRO 4. Tasas de prevalencia, incidencia y abandono del consumo de las principales drogas lícitas. ${ }^{a}$ Chile, 1998

\begin{tabular}{lccccc}
\hline Tipo de droga & $\mathrm{PV}^{\mathrm{b}}$ & $\mathrm{PA}^{\mathrm{c}}$ & $\mathrm{PM}^{\mathrm{d}}$ & Incidencia $^{\mathrm{e}}$ & $\begin{array}{c}\text { Abandono del } \\
\text { consumo }^{\dagger}\end{array}$ \\
\hline Ansiolíticos & 28,35 & 12,52 & 6,89 & 6,75 & 55,54 \\
Alcohol & $(27,62-29,08)$ & $(12,01-13,03)$ & $(6,50-7,28)$ & $(6,32-7,18)$ & $(54,13-56,95)$ \\
& 84,36 & 70,84 & 52,06 & 22,04 & 16,00 \\
Tabaco & $(83,63-85,09)$ & $(70,04-71,64)$ & $(51,20-52,92)$ & $(20,47-23,61)$ & $(15,31-16,69)$ \\
& 71,94 & 47,09 & 40,92 & 9,15 & 34,50 \\
& $(71,18-72,70)$ & $(46,29-47,89)$ & $(40,12-41,72)$ & $(8,33-9,97)$ & $(33,52-35,48)$ \\
\hline
\end{tabular}

a Todos los datos se presentan como porcentajes, con sus intervalos de confianza de $95 \%$.

b $\mathrm{PV}=$ prevalencia a lo largo de toda la vida

c PA = prevalencia en el año anterior a la realización de la encuesta.

d PM = prevalencia en el mes anterior a la realización de la encuesta.

e Incidencia en el año anterior a la realización de la encuesta.

† Abandono del consumo más de un año antes de la realización de la encuesta.

PA según el sexo. El consumo de drogas lícitas en el último año fue, en general, más semejante en ambos sexos que el consumo de drogas ilícitas. Sin embargo, se observaron importantes diferencias según el tipo de droga. Así, mientras el consumo de alcohol en los hombres fue casi 16 puntos porcentuales mayor que en las mujeres y el consumo de tabaco 11 puntos mayor, el consumo de ansiolíticos mostró la tendencia contraria, con 8 puntos porcentuales de diferencia a favor de las mujeres.

PA según la edad. El tabaco y el alcohol mostraron un comportamiento muy similar, registrándose las cifras más elevadas en el grupo de 19 a 25 años. El menor consumo correspondió al grupo de menor edad (12 a 18 años) en el caso del alcohol, y al de mayor edad (45-64 años) en el caso del tabaco. En cambio, el consumo de ansiolíticos aumentó con la edad, registrándose la cifra más elevada en el grupo de 45-64 años.

PA según el nivel socioeconómico. Los resultados fueron diferentes según el tipo de droga. Así, en el caso del alcohol se observó un aumento del consumo a medida que aumentaba el nivel socioeconómico. En cambio, el consumo de tabaco mostró la tendencia contraria. Algo diferente ocurrió con el consumo de ansiolíticos, que mostró prevalencias muy similares en todos los niveles socioeconómicos, aunque la cifra más elevada correspondió al nivel alto.

PM según el sexo. Para el consumo tanto de alcohol como de tabaco, las mayores $\mathrm{PM}$ se registraron en el sexo masculino, en el que el consumo de alcohol fue 20 puntos porcentuales mayor que en las mujeres, y el de tabaco 12 puntos mayor. En cambio, el consumo de ansiolíticos en el sexo femenino triplicó al del sexo masculino.

PM según la edad. Mientras que en el caso del alcohol la prevalencia aumentó hacia el grupo de 19 a 25 años y descendió posteriormente, en el caso del consumo de tabaco se observó una distribución en forma de " $\mathrm{M}$ ". También fue distinta la tendencia observada en el caso del consumo de ansiolíticos, cuya PM aumentó con la edad. El consumo de estas drogas en el grupo de mayor edad fue más de seis veces mayor que el observado en las personas de menos edad. El grupo de 12 a 18 años fue el que presentó las menores tasas de consumo de cualquiera de las tres drogas lícitas consideradas.

PM según el nivel socioeconómico. Las tendencias fueron distintas para cada una de las tres drogas. Mientras que el consumo de alcohol tendió a aumentar hacia las categorías de mayor nivel socioeconómico, el de tabaco mostró la tendencia contraria. El consumo de ansiolíticos mostró una distribución irregular.

Edad de inicio del consumo. Exceptuando el caso de los ansiolíticos, la edad de inicio del consumo de drogas lícitas fue menor que la edad de inicio del consumo de drogas ilícitas. Considerando el percentil 50, mientras el consumo de tabaco se inició a los 15 años y el de alcohol a los 17, el de ansiolíticos comenzó a los 30. Para el percentil 5, las cifras correspondientes fueron de 11, 10 y 13 años, y para el percentil 95 de 25, 25 y 55 años, respectivamente.

\section{Tendencias del consumo de drogas en los estudios de 1994, 1996 y 1998}

A partir de los datos proporcionados por los tres estudios nacionales sobre el consumo de drogas realizados en 1994, 1996 y 1998, en este apartado se analizan, primero, las características de las muestras utilizadas en cada estudio y después las principales tendencias del consumo de drogas en Chile.

Muestras y poblaciones representadas en los tres estudios. Como puede observarse en el cuadro 5, los tamaños muestrales han aumentado a medida que los estudios se han ido centrando en una partición más específica del territorio representado: zonas geográficas censales en 1994, regiones en 1996 y comunas en 1998. El tamaño de la población representada en los diferen-

CUADRO 5. Muestras y poblaciones representadas en los estudios de 1994, 1996 y 1998. Chile

\begin{tabular}{lrrr}
\hline & \multicolumn{1}{c}{1994} & \multicolumn{1}{c}{1996} & \multicolumn{1}{c}{1998} \\
\hline Muestra $(n)$ & 8271 & 12421 & 31665 \\
Población $(N)$ & 6186528 & 6917182 & 6940727 \\
\hline
\end{tabular}


CUADRO 6. Distribución porcentual de las muestras de los estudios de 1994, 1996 y 1998 según el sexo, la edad y el nivel socioeconómico. Chile

\begin{tabular}{lrrr}
\hline \multicolumn{1}{c}{ Variable } & 1994 & 1996 & 1998 \\
\hline Sexo & & & \\
$\quad$ Masculino & 46,6 & 45,5 & 46,0 \\
Femenino & 53,4 & 55,5 & 54,0 \\
Edad (años) & & & \\
12-18 & 17,2 & 19,7 & 17,9 \\
19-25 & 20,0 & 17,4 & 16,9 \\
$26-34$ & 22,4 & 20,5 & 20,1 \\
35-44 & 17,3 & 19,9 & 24,7 \\
45-64 & 23,1 & 22,5 & \\
Nivel socioeconómico & & & 9,6 \\
Alto & 5,2 & 5,3 & 25,0 \\
Medio-alto & 15,9 & 18,8 & 35,4 \\
Medio & 38,4 & 39,6 & 4,0 \\
Medio-bajo & 36,5 & 33,1 & \\
Bajo & 4,0 & 3,3 & \\
\end{tabular}

tes estudios es similar, dado que los tres tuvieron la finalidad de representar al universo urbano de estas particiones territoriales, con la idea de estimar los parámetros para la población de 12 a 64 años de edad.

Como se puede observar en el cuadro 6, las muestras de los tres estudios fueron muy similares en lo que respecta a la distribución por sexos. La distribución por grupos de edad también fue relativamente uniforme, tanto en cada muestra como entre ellas. En cuanto al nivel socioeconómico, los dos primeros estudios muestran una distribución muy similar, pero en el tercero se observa un desplazamiento hacia los niveles socioeconómicos más altos, en detrimento del medio y, sobre todo, del medio-bajo.

Tendencias del consumo de drogas ilícitas. Las tasas de PA proporcionadas por los tres estudios fueron muy similares, con diferencias entre ellos inferiores a un punto porcentual para cualquiera de las tres drogas. Tanto para cualquiera de las tres drogas como para la pasta base y el clorhidrato de cocaína, se observó una ligera tendencia a la disminución de la prevalencia en el estudio de 1996. En cambio, en el caso de la marihuana hubo una ligera tendencia al alza. La PM mostró una tendencia similar, con cambios que nunca superaron un punto porcentual (cuadro 7).

Tendencias del consumo de drogas lícitas. La PA del consumo de ansiolíticos aumentó ligeramente de un estudio para otro y la del consumo de tabaco aumentó en 1996, pero volvió en los estudios de 1994, 1996 y 1998. Chile en los estudios de 1994, 1996 y 1998. Chile a disminuir, aunque ligeramente, en 1998. Para el alcohol, la diferencia entre 1994 y 1998 fue notable (casi 10 puntos porcentuales), pero para los ansiolíticos fue tan solo de algunas décimas. La PM del consumo de alcohol también mostró una tendencia sostenida al alza, con más de 12 puntos porcentuales de diferencia entre 1994 y 1998. En cambio, para el consumo de tabaco se mantuvo prácticamente constante, mientras que para el consumo de ansiolíticos disminuyó entre 1994 y 1996 y volvió a aumentar en 1998, superando ligeramente la cifra de 1994 (cuadro 8).

\section{DISCUSIÓN}

Con este estudio nuestro país es uno de los tres países latinoamericanos que disponen de mediciones estadísticas repetidas en el tiempo con metodología que permite analizar las tendencias del consumo de drogas. Esto, sumado a la edición de un informe anual de la situación del consumo de drogas en Chile, nos sitúa en un excelente nivel de conocimiento y vigilancia de este complejo problema.

CUADRO 7. Prevalencia del consumo de drogas ilícitas en el último año y en el último mes

\begin{tabular}{|c|c|c|c|c|c|c|}
\hline \multirow[b]{2}{*}{ Droga } & \multicolumn{3}{|c|}{$\mathrm{PA}^{\mathrm{a}}$} & \multicolumn{3}{|c|}{$\mathrm{PM}^{\mathrm{b}}$} \\
\hline & 1994 & 1996 & 1998 & 1994 & 1996 & 1998 \\
\hline Marihuana & 4,00 & 4,01 & 4,73 & 1,84 & 1,20 & 1,95 \\
\hline Pasta Base & 0,86 & 0,63 & 0,81 & 0,33 & 0,21 & 0,37 \\
\hline Cocaína & 0,90 & 0,83 & 1,32 & 0,31 & 0,25 & 0,41 \\
\hline Cualquiera & 4,45 & 4,26 & 5,31 & 2,02 & 1,36 & 2,23 \\
\hline
\end{tabular}

a PA = prevalencia en el año anterior a la realización de la encuesta.

${ }^{\mathrm{b}} \mathrm{PM}=$ prevalencia en el mes anterior a la realización de la encuesta.

CUADRO 8. Prevalencia del consumo de drogas lícitas en el último año y en el último mes

\begin{tabular}{lrrrrrrr}
\hline & \multicolumn{3}{c}{ PA $^{\mathrm{a}}$} & & \multicolumn{3}{c}{ PM $^{\mathrm{b}}$} \\
\cline { 2 - 3 } \cline { 6 - 7 } Droga & 1994 & 1996 & 1998 & & 1994 & 1996 & 1998 \\
\hline Ansiolíticos & 12,07 & 12,22 & 12,52 & & 6,20 & 5,64 & 6,89 \\
Alcohol & 60,61 & 70,25 & 70,84 & & 39,97 & 46,66 & 52,06 \\
Tabaco & 45,77 & 47,54 & 47,09 & & 40,53 & 40,37 & 40,92 \\
\hline
\end{tabular}

a $P A=$ prevalencia en el año anterior a la realización de la encuesta.

b $\mathrm{PM}=$ prevalencia en el mes anterior a la realización de la encuesta. 
Tanto en el estudio de 1998 como en los anteriores, las cifras encontradas pueden considerarse moderadas para el consumo de drogas ilícitas, lo cual probablemente es una consecuencia de las políticas y acciones que en conjunto representan la respuesta del país en este tema. Sin embargo, hay que reconocer que es probable que las cifras registradas en este estudio subestimen las cifras reales. A pesar de que las tasas de consumo de drogas ilícitas son bajas, se ha observado un ligero aumento entre 1996 y 1998, explicado básicamente por el aumento del consumo de marihuana. En el caso de las drogas lícitas la situación es diferente. Las tasas de consumo de alcohol y tabaco en el último año son elevadas, pero muestran una tendencia a la estabilización en el tiempo. El consumo de ansiolíticos en el último año, siendo casi la sexta parte del consumo de alcohol, muestra una tendencia estable con leves alzas desde 1994. Aunque podrían haberse esperado disminuciones del consumo por modificaciones de la legislación sobre estos fármacos, la estabilización de las cifras podría ser consecuencia de la sustitución de ciertos fármacos por otros menos controlados, como los llamados "naturales".

El consumo de drogas ocurre en todo el país, en ambos sexos, en todos los grupos de edad y en todos los niveles socioeconómicos, aunque existen diferencias entre los diferentes grupos que son las que permiten establecer prioridades para los distintos programas y proyectos de prevención.

Tomando como referencia la prevalencia del consumo de drogas ilícitas en el último año, dicho consumo fue mayor en el sexo masculino, entre los 19 y 25 años y en el nivel socioeconómico bajo. Considerando las edades más afectadas por el consumo de drogas ilícitas, las instituciones de educación superior, como las universidades, institutos profesionales y centros de formación técnica, y otras instituciones que atienden a los individuos de ese grupo de edad se enfrentan al reto de crear programas que posibiliten la prevención y tratamiento de la drogadicción entre las poblaciones atendidas.

Mientras que el consumo de marihuana y de clorhidrato de cocaína está presente en todas las categorías de nivel socioeconómico, el consumo de pasta base es mayor en el nivel socioeconómico más bajo. Estos datos sugieren la necesidad de incorporar el tema de la prevención del consumo de esta droga a todos los programas destinados a la superación de la pobreza $\mathrm{y}$, en general, a las acciones de las instituciones públicas y privadas que en el país atienden a los sectores de población más desfavorecidos. El fundamento para ello se basa en la falta de estructuras, círculos de influencia y medios económicos para enfrentar la situación de enfermo de quien ha desarrollado dependencia, y también en la falta de oportunidades que hace que la droga sea parte del proyecto de vida de quien la usa inicialmente con fines exploratorios o para suplir carencias de alimento y afecto.

El incremento del consumo de marihuana fue proporcionalmente mayor en las mujeres que en los hombres. Entre 1994 y 1998 la prevalencia del consumo de marihuana en el último año aumentó en las mujeres en 33\% (de 1,8 a 2,4\%), mientras que en los hombres solo aumentó en 15\% (de $6,5$ a $7,5 \%)$. Con la cocaína ocurrió lo mismo, aunque la diferencia entre los sexos fue menor. En las mujeres aumentó en $66 \%$ (de 0,3 a $0,5 \%$ ), mientras que en los hombres aumentó en $44 \%$ (de 1,6 a $2,3 \%$ ).

Las tendencias del consumo según la edad muestran que son los jóvenes los que más han incrementado el consumo de drogas ilegales. En el caso de la marihuana, el mayor aumento de la prevalencia en el último año correspondió al grupo de 12 a 18 años: de 5,5 a $7,8 \%$, lo cual representa un aumento de $42 \%$. Para la cocaína, el mayor aumento correspondió al grupo de 19 a 25 años: de 1,8 a 3,4\%, lo cual representa un aumento de $89 \%$.

En cuanto a la tendencia en los distintos niveles socioeconómicos, lo más destacable es el incremento del consumo de cocaína en los niveles socioeconómicos medio-bajo y bajo. En el nivel medio-bajo la prevalencia en el último año pasó de 0,3 a 1,3\%, lo cual representa un aumento de $333 \%$, y en el nivel bajo de 0,3 a $1,4 \%$, lo cual representa un aumento de $367 \%$.

\section{REFERENCIAS}

1. Chile, Ministerio del Interior, Consejo Nacional para el Control de Estupefacientes. Política y Plan Nacional de Prevención y Control de Drogas. Santiago de Chile: Ministerio del Interior; 1993.

2. Fuentealba R, Cumsille F, Molina C, Rojas F, Hintze G, Varela T. Consejo Nacional para el Control de Estupefacientes. Informe final del Primer Estudio Nacional de Consumo de Drogas. Santiago de Chile: Ministerio del Interior; 1996.

3. Fuentealba R, Molina C, Cumsille F, Calderón R, Araneda J, Varela T. Consejo Nacional para el Control de Estupefacientes. Segundo Estudio Nacional de Consumo de Drogas en Chile. Santiago de Chile: Ministerio del Interior; 1998.

4. Fuentealba R, Cumsille F, Molina C, Araneda J, Calderón R, Varela T. Consejo Nacional para el Control de Estupefacientes. Informe ejecutivo del Tercer Estudio Nacional de Consumo de Drogas. Santiago de Chile: Ministerio del Interior; 1999.

5. Torres Y, Montoya I. Segundo Estudio de Salud Mental y Consumo de Sustancias Psicoactivas. Santa Fe de Bogotá: Ministerio de Salud de Colombia; 1997.
6. Díaz E. Clasificación socioeconómica internacional. Pediatría (Santiago de Chile) $1975 ; 18$.

7. Florenzano R, Gazmuri C, Carrasco E. Alcoholismo y farmacodependencias juveniles en Chile. 1a ed. Santiago de Chile: CPU; 1992.

8. Cortés F, Reinoso A, Urrutia L. Instituto Nacional de la Juventud. Encuesta de Juventud Gran Santiago. Informe de resultados. Santiago de Chile: Ministerio de Planificación y Cooperación; 1992.

9. Molina C, Heras R, Ortiz C, Arancibia S, Soto F, Villarroel R, et al. Diagnóstico de la situa- 
ción del alcoholismo y la drogadicción en niños y jóvenes de la Educación General Básica y Media de la Cuarta Región Coquimbo. Santiago de Chile: Ministerio de Educación (CPEIP); 1982-1986. (Serie de Estudios Nos. 76, 86, 89, 97, 101, 130 y 133).
10. Corporación Programa de Atención para Drogadictos "Caleta Sur". Diagnóstico sobre drogadicción en poblaciones Santa Mónica y Villa Araucanía. Informe del Programa Poblacional de Servicios para Drogadictos Caleta Norte. Santiago de Chile: Programa Poblacional de Servicios para Drogadictos Caleta Norte; 1990.
Manuscrito recibido el 21 de agosto de 1998 y aceptado para publicación, tras revisión, el 13 de septiembre de 1999.

ABSTRACT The principal results are presented here from the Third National Study of the Consumption of Drugs, which was carried out in Chile by the National Board for Narcotics Control between 1 September 1998 and 15 January 1999. The 1998 study used the same methodological design as the first and second studies, which were done in 1994 and 1996. The 1998 study expanded the sample to 31665 individuals, who were representative of a population of 6940727 people from 12 to 64 years old, both sexes, and five socioeconomic levels and who were residents of urban areas in 62 commune administrative divisions of the 13 regions of the country. Results from the 1998 study are compared with those from 1996 and 1994.

The 1998 study shows that $17.5 \%$ of Chileans have at some time in their life used one of the three illicit drugs most popular in the country: marijuana (16.8\%), coca paste $(2.3 \%)$, and cocaine hydrochloride $(4.0 \%)$. The prevalence of use of any of the three drugs, mainly marijuana, during the preceding year was $5.3 \%$ and during the preceding month $2.2 \%$. With respect to licit drugs, $28.4 \%$ of Chileans have at some time in their lives used antianxiety drugs, $84.4 \%$ of them have consumed alcohol, and $71.9 \%$ have used tobacco. Most of the people who once used illegal drugs have stopped doing so: $71.6 \%$ in the case of marijuana, $64.1 \%$ with coca paste, and $66.8 \%$ with cocaine hydrochloride. For legal drugs, the percentages were lower: $55.5 \%$ for anxiolytics, $16.0 \%$ for alcohol, and $34.5 \%$ for tobacco.

The consumption of licit and illicit drugs was several times greater among men than among women, except for anxiolytics, whose use was three times greater among women. Drug use was more frequent among persons between 19 and 25 years old. Consumption of illegal drugs was more frequent at higher socioeconomic levels, and use of licit drugs was more common in the lower socioeconomic levels. The 50th percentile of the age of initiating drug use was 17 years for alcohol, 15 for tobacco, 30 for antianxiety agents, 17 for marijuana, 20 for coca paste, and 21 for cocaine hydrochloride.

Comparing the results of the three studies shows that, after an increase in the use of licit drugs between 1994 and 1996, there was a stabilization in 1998. With illegal drugs there was a modest increase in consumption between 1996 and 1998, following a small reduction between 1994 and 1996. 